\title{
DNA polymerases beta and lambda: gap-filling synthesis on the intact and damaged DNA templates in base excision repair
}

\author{
E.A. Maltseva, L.V. Starostenko, O.I. Lavrik, N.I. Rechkunova \\ Institute of Chemical Biology and Fundamental Medicine SB RAS, Novosibirsk, Russia
}

Key words: base excision repair, cluster lesions, DNA polymerases, PARP1

Motivation and Aim: DNA polymerases beta and lambda (Pol $\beta$ and Pol $\lambda$ ) belong to the $\mathrm{X}$-family and possess similar activities. Both enzymes have dRP lyase activity and the ability to effectively foster gap-filling reactions. We showed earlier that under certain conditions these DNA polymerases can catalyze lesion bypass across benzo[a]pyrenederived (BP-dG) DNA adduct during repair of apurinic/apyrimidinic (AP) sites [1]. Cluster-type DNA damages that include both a modified nucleotide and a bulky lesion can be generated under combined action of oxidative stress and genotoxic polycyclic aromatic hydrocarbons derivatives. In the present study we analyzed the ability of DNA polymerases $\beta$ and $\lambda$ to fil gaps generated in DNA after cleavage of AP sites located in different positions to the BP-dG adduct and their interaction with AP-site cleaving enzyme - AP endonuclease 1 (APE1). Effect of DNA repair regulating protein poly(ADP-ribose)polymerase 1 (PARP1) on Pol $\beta$ and Pol $\lambda$ activities was also studied.

Methods: Biochemical and immunochemical approaches, fluorescence titration methods, molecular dynamic simulation.

Results: The activity of Pol $\beta$ and Pol $\lambda$ depends on the location of the gap relative to the modified nucleotide as well as on the conformation of the bulky adduct and on the reaction conditions. DNA duplex with the AP site in $3^{\prime}$ adjacent $(+1)$ position to nucleotide paired with the modified $\mathrm{dG}$ was the most difficult to be processed by APE1 and DNA polymerases. The repair process was more effective when both APE1 and Pol $\beta$ were added simultaneously to this DNA. No functional cooperation between APE1 and Pol $\lambda$ was observed. Pol $\beta$ was also able to insert the correct nucleotide in the gap arising after AP site hydrolysis in DNA with the AP site in position +2 and +3 . Pol $\lambda$ was more sensitive to stereoisomeric form of BP-dG adducts and performed DNA synthesis with less fidelity than Pol $\beta$. Although PARP1 moderately inhibited both DNA polymerases, in the presence of NAD + only Pol $\beta$ activity was completely restored.

Conclusion: Pol $\beta$ tolerates BP-dG adducts in DNA and in cooperation with APE1 can correctly process AP site clustered with bulky lesion. Pol $\lambda$ is sensitive to stereoisomeric form of BP-dG adducts and to PARP1 catalyzed modification and can play a specific role in the repair of cluster lesions.

Acknowledgements: This work was supported by RFBR grant No. 18-04-00596.

\section{References}

1. Starostenko L.V., Rechkunova N.I., Lebedeva N.A., Kolbanovskiy A., Geacintov N.E., Lavrik O.I. (2014) Human DNA polymerases catalyze lesion bypass across benzo[a]pyrene-derived DNA adduct clustered with an abasic site. DNA Repair. 24:1-9. 